S. Shirai

Nagoya Math. J.

Vol. 71 (1978), 87-90

\title{
A REMARK CONCERNING THE 2-ADIC NUMBER FIELD
}

\author{
SUSUMU SHIRAI
}

\section{Introduction}

Let $Q_{2}$ be the 2-adic number field, $T / Q_{2}$ be a finite unramified extension, $\zeta_{\nu}$ be a primitive $2^{\nu}$-th root of unity, and let $K_{\nu}=T\left(\zeta_{\nu}\right)$. In a previous paper [1, Theorem 11], we stated the following theorem without its proof.

THEOREM A. Let $R=T\left(\zeta_{\nu}+\zeta_{\nu}^{-1}\right)$, and let $\sigma$ be a generator of the cyclic Galois group $G(R / T)$. Assume $\nu \geqq 3$. If $N_{R / T} \varepsilon=1$ for $\varepsilon \in U_{R}^{(4)}$, then

$$
\varepsilon \in\left(N_{K_{\nu} / R} K_{\nu}^{\times}\right)^{\sigma-1},
$$

where $U_{R}^{(i)}$ denotes the $i$-th unit group of $R$.

The aim of the present paper is to prove this theorem, which is a detailed version of Hilbert's theorem 90 in the 2-adic number field.

\section{Preliminaries}

Let $\theta=\zeta_{\nu}+\zeta_{\nu}^{-1}$. Since $1-\zeta_{\nu}$ is a prime element of $K_{\nu}$,

$$
N_{K_{\nu} / R}\left(1-\zeta_{\nu}\right)=\left(1-\zeta_{\nu}\right)\left(1-\zeta_{\nu}^{-1}\right)=2-\theta
$$

is a prime element of $R$. Set $\pi=2-\theta$ and denote by $\nu_{\pi}$ the normalized exponential valuation of $R$. The Galois group $G\left(K_{\nu} / T\right)$ is isomorphic to the group of prime residue classes $\bmod 2^{\nu}$, and hence we can choose the generator $\sigma$ of $G(R / T)$ such that

$$
\theta^{\sigma}=\left(\zeta_{\nu}+\zeta_{\nu}^{-1}\right)^{\sigma}=\zeta_{\nu}^{5}+\zeta_{\nu}^{-5}=\theta^{5}-5 \theta^{3}+5 \theta,
$$

without loss of generality. Then

$$
\pi^{\sigma}=\pi^{5}-10 \pi^{4}+35 \pi^{3}-50 \pi^{2}+25 \pi .
$$

LEMMA 1. Notation being as above, if $\nu \geqq 3$, then

Received June 10, 1977. 


$$
\nu_{\pi}\left(\pi^{o}-\pi\right)=3
$$

Proof. Immediate from (1).

LEMMA 2. If $\nu \geqq 3$, then

$$
\nu_{x}\left(\left(\pi^{n}\right)^{\sigma-1}-1\right) \begin{cases}=2 & \text { when } n \text { is odd }, \\ \geqq 4 & \text { when } n \text { is even } .\end{cases}
$$

Proof. By Lemma 1, we have

$$
\nu_{\pi}\left(\pi^{\sigma-1}-1\right)=2,
$$

and hence we can write

$$
\pi^{o-1}=1+a \pi^{2}, \quad(a, \pi)=1 .
$$

Therefore, for $n \geqq 1$,

$$
\left(\pi^{n}\right)^{o-1}-1=\pi^{2}\left(n a+n(n-1) / 2 \cdot a^{2} \pi^{2}+\cdots\right) .
$$

We have $\nu_{\pi}\left(\left(\pi^{n}\right)^{\sigma-1}-1\right)=2$ if $n$ is odd. Since $\nu_{x}(2)=2^{\nu-2} \geqq 2$, we have $\nu_{\pi}\left(\left(\pi^{n}\right)^{o-1}-1\right) \geqq 4$ if $n$ is even. For $n \leqq-1$, according as $n$ is odd or even, we obtain

$$
\left(\pi^{-n}\right)^{\sigma-1} \in U_{R}^{(2)}-U_{R}^{(3)} \quad \text { or } \quad \in U_{R}^{(4)}
$$

This completes the proof.

LEMMA 3. If $\nu \geqq 3$, then

$$
\nu_{\pi}\left(\beta^{o-1}-1\right) \geqq 4 \quad \text { for } \beta \in U_{R}^{(2)} .
$$

Proof. We may write

$$
\beta=1+a \pi^{2}, \quad a \in O_{R} \text {, the ring of integers of } R .
$$

Then

$$
\beta^{\sigma-1}-1=\left(a^{\sigma}\left(\pi^{\sigma}\right)^{2}-a \pi^{2}\right) / \beta .
$$

Since $R / T$ is totally ramified, $\left\{1, \pi, \cdots, \pi^{2 \nu-2-1}\right\}$ is an integral basis for $R / T$. Set

$$
a \equiv a_{0}+a_{1} \pi+a_{2} \pi^{2}+a_{3} \pi^{3} \quad \bmod \pi^{4}, a_{i} \in O_{T}
$$

Then

$$
a^{\sigma} \equiv a_{0}+a_{1} \pi^{\sigma}+a_{2}\left(\pi^{\sigma}\right)^{2}+a_{3}\left(\pi^{\sigma}\right)^{3} \quad \bmod \pi^{4} .
$$

By (1) and $\nu_{\pi}(50)=2^{\nu-2} \geqq 2$, we have 


$$
\pi^{\sigma} \equiv 35 \pi^{3}+25 \pi \quad \bmod \pi^{4}
$$

Hence

$$
\begin{aligned}
a^{\sigma}\left(\pi^{o}\right)^{2}-a \pi^{2} & \equiv 624 a_{0} \pi^{2}+15624 a_{1} \pi^{3} \\
& =2^{4} \cdot 3 \cdot 13 a_{0} \pi^{2}+2^{3} \cdot 3^{2} \cdot 7 \cdot 31 a_{1} \pi^{3} \\
& \equiv 0 \quad \bmod \pi^{4} .
\end{aligned}
$$

Next, let $\left[T: Q_{2}\right]=f$, and let $\xi$ be a primitive $\left(2^{f}-1\right)$ st root of unity. It is well-known that $T=Q_{2}(\xi)$ and $\left\{1, \xi, \cdots, \xi^{f-1}\right\}$ is an integral basis for $T / Q_{2}$, and moreover $U_{R}^{(1)} / U_{R}^{(2)} \approx \bar{R}=\bar{T}$ is a module of type $\underbrace{(2, \cdots, 2)}_{f}$, where $\bar{R}, \bar{T}$ stand for the residue class fields of $R$ and $T$, respectively. As a complete system of representatives for $U_{R}^{(1)} / U_{R}^{(2)}$, we can choose

$\left\{\gamma=(1+\pi)^{n_{0}}(1+\xi \pi)^{n_{1}} \cdots\left(1+\xi^{f-1} \pi\right)^{n_{f-1}} ; n_{i}=0\right.$ or $\left.1, i=0,1, \cdots, f-1\right\}$.

Lemma 4. Notation being as above, if $\nu \geqq 3$ and $\gamma \neq 1$, then

$$
\nu_{\pi}\left(\gamma^{\sigma-1}-1\right)=3 \text {. }
$$

Proof. Since

$$
\gamma=\left(1+n_{0} \pi\right)\left(1+n_{1} \xi \pi\right) \cdots\left(1+n_{f-1} \xi^{f-1} \pi\right),
$$

we have

$$
\begin{aligned}
\gamma^{\sigma}-\gamma= & \left(\pi^{\sigma}-\pi\right)\left(n_{0}+n_{1} \xi+\cdots+n_{f-1} \xi^{f-1}\right) \\
& +\left(\left(\pi^{\sigma}\right)^{2}-\pi^{2}\right)(\cdots \cdots) \\
& +\cdots .
\end{aligned}
$$

From Lemma 1, we obtain

$$
\nu_{\pi}\left(\pi^{\sigma}-\pi\right)=3, \quad \nu_{\pi}\left(\left(\pi^{\sigma}\right)^{2}-\pi^{2}\right) \geqq 4, \cdots .
$$

Thus it suffices to show that

$$
n_{0}+n_{1} \xi+\cdots+n_{f-1} \xi^{f-1} \not \equiv 0 \quad \bmod \pi .
$$

Suppose $\equiv 0 \bmod \pi$. Then we have

$$
n_{0}+n_{1} \xi+\cdots+n_{f-1} \xi^{f-1} \equiv 0 \quad \bmod \pi_{T},
$$

$\pi_{T}$ being a prime element of $T$. Since $\left\{\xi^{i} \bmod \pi_{T} ; i=0,1, \cdots, f-1\right\}$ is a basis of the residue class field extension $\bar{T} / \bar{Q}_{2}$, we conclude all 
$n_{i}=0$, a contradiction

\section{Proof of Theorem $A$}

We first note that

$$
\pi=2-\theta=N_{K_{\nu} / R}\left(1-\zeta_{\nu}\right) \in N_{K_{\nu} / R} K_{\nu}^{\times}, \quad \xi \in N_{K_{\nu} / R} K_{\nu}^{\times}, \quad U_{R}^{(2)} \subset N_{K_{\nu} / R} K_{\nu}^{\times},
$$

in which the second follows from that the order $2^{f}-1$ of $\xi$ is prime to $\left[R^{\times}: N_{K_{\nu} / R} K_{\nu}^{\times}\right]=2$ and the third from that the $\pi$-exponent of the conductor of $K_{\nu} / R$ is two. Now, let $\varepsilon$ be an element in $U_{R}^{(4)}$ such that $N_{R / T} \varepsilon=1$. Then we can write, by Hilbert's theorem 90 ,

$$
\varepsilon=\alpha^{\sigma-1}, \quad \alpha \in R^{\times} .
$$

Since $R^{\times}=\langle\pi\rangle \times\langle\xi\rangle \times U_{R}^{(1)}$ (a direct product) and $U_{R}^{(1)} \supset U_{R}^{(2)}$, we may set

$$
\alpha=\pi^{n} \cdot \xi^{m} \cdot \gamma \cdot \beta, \quad \beta \in U_{R}^{(2)},
$$

here $\gamma$ is as in Lemma 4. By virtue of the above remark, it completes the proof that we obtain $\gamma=1$. Assume $\gamma \neq 1$. Then we have

$$
\varepsilon=\left(\pi^{n}\right)^{\sigma-1} \cdot \gamma^{\sigma-1} \cdot \beta^{\sigma-1},
$$

in which Lemmas 3,4 give $\beta^{\sigma-1} \in U_{R}^{(4)}$ and $\gamma^{\sigma-1} \in U_{R}^{(3)}-U_{R}^{(4)}$, respectively. If $n$ is even, then we have, by Lemma $2,\left(\pi^{n}\right)^{\sigma-1} \in U_{R}^{(4)}$, a contradiction. If $n$ is odd, then we have, by Lemma $2,\left(\pi^{n}\right)^{\sigma-1} \in U_{R}^{(2)}-U_{R}^{(3)}$ from which follows $\left(\pi^{n}\right)^{\sigma-1} \cdot \gamma^{\sigma-1} \in U_{R}^{(2)}-U_{R}^{(3)}$, a contradiction, and the proof is complete.

\section{REFERENCE}

[1] S. Shirai, On the central class field $\bmod \mathfrak{m}$ of Galois extensions of an algebraic number field, Nagoya Math. J., 71 (1978), 61-85.

Toyama Medical and Pharmaceutical University 